\title{
Thrombospondin-1 Short Hairpin RNA Suppresses Tubulointerstitial Fibrosis in the Kidney of Ureteral Obstruction by Ameliorating Peritubular Capillary Injury
}

\author{
Dong Sun ${ }^{\mathrm{a}, \mathrm{b}}$ Yanyan Ma ${ }^{\mathrm{a}}$ Hong Han ${ }^{\mathrm{a}}$ Zhongcheng Yin ${ }^{\mathrm{a}}$ Caixia Liu ${ }^{\mathrm{a}}$ \\ Jinhong Feng ${ }^{a}$ Xudong Zhou $^{\mathrm{b}}$ Xiaoju $\mathrm{Li}^{\mathrm{b}}$ Aiguo Xiao $^{\mathrm{b}}$ Rong Yu ${ }^{\mathrm{b}}$ \\ a Department of Nephrology, Affiliated Hospital of Xuzhou Medical College, Xuzhou, and \\ ${ }^{b}$ Department of Nephrology, Kuitun Hospital of Yili State, Kuitun, PR China
}

\section{Key Words}

Thrombospondin-1 - Short hairpin RNA - Renal interstitial fibrosis - Unilateral ureteral occlusion - Peritubular capillary density

\begin{abstract}
Background/Aims: Thrombospondin-1 (TSP-1), a naturally occurring inhibitor of angiogenesis, is an important mediator of renal fibrosis in clinical and experimental kidney disease. Increasing evidence shows that the microvasculature plays a critical role in progressive renal disease. The present study was undertaken to investigate whether interstitial fibrosis could be prevented by abolishing TSP-1 function in unilateral ureteral obstruction (UUO)-induced renal fibrosis. Methods and Results: A short hairpin RNA vector, designated Thbs-1, significantly suppressed TSP-1 in both transcriptional and translational levels in in vitro-cultured cells and in vivo fibrosis-induced mouse kidney. Furthermore, TSP-1 RNA interference increased the protein level of vascular endothelial growth factor (VEGF) and the density of peritubular capillaries (PTCs), reduced the expression of hypoxia-inducible factor- $1 \alpha$ in tubulointerstitial cells, and collagen III and the connective tissue growth factor expression were markedly reduced from day 7 after UUO-induced fibrosis, but un-
\end{abstract}

or vector-treated mice maintained their expression. TSP-1 shRNA suppressed the protein level of TSP-1, increased VEGF expression and PTC density and alleviated the development of renal interstitial fibrosis in UUO mice. Conclusion: These data suggest that inhibition of TSP-1 expression prevented tubulointerstitial fibrosis through ameliorating PTC injury.

Copyright $\odot 2011$ S. Karger AG, Basel

\section{Introduction}

Renal fibrosis is regarded as the final common pathway for most forms of progressive renal disease, and it involves glomerular sclerosis and/or interstitial fibrosis [1]. It has been shown that tubulointerstitial injury is a more consistent predictor of functional impairment than glomerular damage in a number of clinical as well as experimental studies $[2,3]$. Exploration of the mechanisms of tubulointerstitial fibrosis is important for establishing new therapeutic approaches for prevention of progressive renal disease. Although many studies have focused on the mechanisms underlying excessive deposition of glomer-

Yanyan Ma and Hong Han should be considered as first co-authors.

\section{KARGER \\ Fax +41613061234 E-Mail karger@karger.ch} www.karger.com
Dr. Dong Sun

Department of Nephrology, Affiliated Hospital of Xuzhou Medical College

99 West Huai-hai Road

Xuzhou, Jiangsu 221002 (PR China)

Tel. +865168580 2174, E-Mail sdxyfy@yahoo.cn 
ular and tubulointerstitial extracellular matrix, a growing body of evidence supports the view that the vascular component is involved in the pathogenesis of renal fibrosis. Loss of peritubular capillaries (PTCs) is associated with progressive interstitial fibrosis [4-8], which would be expected to result in chronic ischemia and hypoxia that could stimulate interstitial fibrosis [9].

Thrombospondin-1 (TSP-1) is a large glycoprotein that is synthesized and secreted by the different types of cells [10]. TSP-1 expression is markedly increased in the interstitium in both experimental $[11,12]$ and human [13] interstitial fibrosis and is a strong predictor of the subsequent development of interstitial fibrosis [11]. TSP-1, which can directly initiate endothelial cell apoptosis [14], is the first protein to be shown to play a critical role as a naturally occurring inhibitor of angiogenesis [15]. There is increasing evidence that TSP-1 expression correlates with a loss of the microvascular endothelium [7]. Thus, the disruption of TSP-1 expression may increase the number of PTCs and alleviate renal fibrosis, which may be an ideal strategy for the inhibition of renal fibrosis.

Antisense oligonucleotide has been widely used for gene regulation in both cell cultures and animal models [16]. However, as a therapeutic tool, antisense oligonucleotide has several problems, such as short retention time and nonspecific activity [17]. Compared with antisense oligonucleotide, RNA interference (RNAi)-based gene silencing shows greater potency and promising specificity. Small interfering RNA (siRNA) and short hairpin RNA (shRNA) are two general approaches to induce RNAi in mammalian cells. Transfection with synthetic siRNA only induces transient silencing. Stable induction of RNAi usually utilizes shRNA expressed from plasmid or viral vectors [18]. Notably, recent studies have revealed that delivery of plasmid DNA expressing shRNA is superior to siRNA because of its effective inhibition [19-21].

In this study, we constructed three plasmids to express individual shRNA and evaluated their activity in rat mesangial cells (RMCs). The most effective shRNA expression plasmid was tested in unilateral ureteral obstruction (UUO) kidney in mice in the shRNA group for gene silencing and therapeutic efficacy. Our results demonstrated that the treatment with TSP-1 shRNA suppressed the expression of TSP-1, while vascular endothelial growth factor (VEGF) and PTCs were increased after TSP-1 shRNA administration in the UUO mouse kidney. The development of tubulointerstitial fibrosis was significantly alleviated in the mouse UUO kidney after TSP-1 shRNA administration.

\section{Materials and Methods}

\section{Construction of TSP-1 shRNAs Vectors}

The shRNA-expressing plasmids, Thbs1-1, -2 or -3 were purchased from the pEGFP vector (Shanghai GeneChem Co., Shanghai, China) and designed to express individual shRNA, driven by the human U6 promoter. They contained three pairs of shRNA sequences targeting the TSP-1 gene (table 1). The rat accession No. of the TSP-1 gene is NM_001013062 and the mouse accession No. of the TSP-1 gene is NM__011580. These plasmids were designed to transcribe a stem-loop-type RNA with loop sequences of TTC AAG AGA.

\section{Cell Culture and Transfection}

RMCs were obtained from Wuhan University Cell Storage Center (Wuhan, China). The cells were grown in Dulbecco's modified Eagle medium (DMEM; GIBCO, Grand Island, N.Y., USA) supplemented with $10 \% \mathrm{FBS}$ (GIBCO) at $37^{\circ} \mathrm{C}$ in a humidified atmosphere of $5 \% \mathrm{CO}_{2}$. Twenty-four hours before transfection, 2 $\times 10^{5}$ cells were seeded in each well of a 6-well plate and cultured in growth medium without antibiotics to achieve $>50 \%$ confluence on the day of transfection. For shRNA transfection, the cells were washed twice with Opti-MEM (Gibco-BRL, USA) and transfected with TSP-1 shRNA vector (table 1) or empty vector with Lipofectamine 2000 (Invitrogen, Carlsbad, Calif., USA). Cells were incubated at $37^{\circ} \mathrm{C}$ for $6 \mathrm{~h}$ and cultured in fresh medium containing a low $(5.5 \mathrm{mM})$ or high $(30 \mathrm{mM})$ concentration of glucose.

Experimental Animal Model and shRNA Delivery

Male Kunming mice weighing approximately 25-30 g were used and divided into four groups: (1) normal control, (2) UUO with no treatment, (3) EV treated with empty vector, and (4) shRNA treated with Thbs-1. The UUO model was performed using an established procedure [22]. In brief, under pentobarbitalinduced anesthesia, the left ureter was ligated with silk (4/0) at two locations and cut between ligatures to prevent urinary tract infection. After undergoing surgery, the shRNA was delivered by injection of $10 \mu \mathrm{g}$ shRNA vector DNA (Thbs-1) or $10 \mu \mathrm{g}$ empty vector (EV) through the renal artery in the In Vivo Gene Delivery System (Mirus) [23]. Six mice of each group were sacrificed at 1, 3, 7 and 14 days after operation, and obstructed kidneys were harvested and subjected to the studies described below. The animals were treated in accordance with the Xuzhou Medical College Animal Center Committee.

\section{$R T-P C R$}

The expression levels of the genes of interest were characterized by RT-PCR using the Reverse Transcriptase-Polymerase Chain Reaction Kit (Bioer, Hangzhou, China), according to the manufacturer's instruction. Briefly, total RNA was extracted from the cells that had been transfected with the plasmid expressing the target or control shRNA and reversely transcribed into cDNA, which was used as the template for the PCR. Aliquots of cDNA were used for PCR using primer sets specific to rat TSP-1 and actin as a control. The specific primers for each gene were synthesized by GeneChem Technology (Shanghai, China) and can be seen in table 2. The PCR reactions were subjected to $94^{\circ} \mathrm{C}$ for 3 min, and then 25 cycles of $94^{\circ} \mathrm{C}$ for $30 \mathrm{~s}, 56^{\circ} \mathrm{C}$ for $30 \mathrm{~s}$ and $72^{\circ} \mathrm{C}$ for $45 \mathrm{~s}$, followed by extension at $72^{\circ} \mathrm{C}$ for $5 \mathrm{~min}$. The PCR products were separated by agarose gel electrophoresis and the expres- 
Table 1. The sequences of shRNA

\begin{tabular}{|c|c|c|c|c|c|}
\hline $\begin{array}{l}\text { Gene } \\
\text { name }\end{array}$ & Stem & Loop & Stem & $\begin{array}{l}\text { Rat target } \\
\text { site }\end{array}$ & $\begin{array}{l}\text { Mouse } \\
\text { target site }\end{array}$ \\
\hline Thbs1-1 & caCCAACTACATCGGCCACAA & TTCAAGAGA & TTGTGGCCGATGTAGTTGGtg & $800-820$ & $950-970$ \\
\hline Thbs1-2 & aaCGCAACCTACCACTGCAAA & TTCAAGAGA & TTTGCAGTGGTAGGTTGCGtt & $2157-2177$ & $2307-2327$ \\
\hline Thbs1-3 & aaCTGTCCATTCCATTACAAC & TTCAAGAGA & GTTGTAATGGAATGGACAGtt & $2292-2312$ & $2442-2462$ \\
\hline
\end{tabular}

Table 2. The sequences of specific primers

\begin{tabular}{lll}
\hline Genes & Sequences & $\begin{array}{l}\text { Expected size } \\
\text { of PCR products }\end{array}$ \\
\hline TSP-1 & $\begin{array}{l}\text { Sense: } 5^{\prime} \text {-CCCTGTGGCATGACCCTCGT-3' } \\
\text { Antisense: 5'-GCCCACACTATGCAAGAAGAGAGG-3' } \\
\text { Actin }\end{array}$ & $\begin{array}{l}\text { Sense: } 5^{\prime} \text {-TCACCCACACTGTGCCCATCTAC-3' } \\
\text { Antisense: } 5^{\prime} \text {-GAGTACTTGCGCTCAGGAGGAG-3' }\end{array}$ \\
\hline
\end{tabular}

sion levels of TSP-1 were semiquantified by densitometric analysis. The relative levels of TSP-1 mRNA transcripts were determined and presented as the ratio of TSP-1 to actin expression.

\section{Western Blot Analysis}

Cells and tissues were lysed in RIPA Lysis Buffer (Bytotime, Wuhan, China) on ice and centrifuged at 12,000 $\mathrm{g}$ for $30 \mathrm{~min}$. Protein samples were electrophoresed through a $7 \%$ polyacrylamide gel and then transferred to nitrocellulose membranes (Amersham SA, France). The membranes were blocked in 5\% skim milk powder for $1 \mathrm{~h}$, and then incubated overnight at $4^{\circ} \mathrm{C}$ with primary polyclonal mouse anti-TSP-1 (1:500), rabbit anti-VEGF (1:100), rabbit anti-hypoxia inducible factor- $1 \alpha$ (HIF- $1 \alpha ; 1: 100$; Santa Cruz, Calif., USA) or rabbit anti-connective tissue growth factor (CTGF; 1:100; Boster, Wuhan, China). The immune complexes were detected using an alkaline phosphatase-conjugated second antibody (Zhongshan, Beijing, China) and a BCIP/NBT alkaline phosphatase color development kit (Beyotime, Shanghai, China). Positive immunoreactive bands were quantified densitometrically and normalized by actin.

\section{Histological Analysis}

Formalin-fixed and paraffin-embedded kidneys tissues were cut into $4-\mu \mathrm{m}$ thick sections. The sections were then stained with hematoxylin-eosin and Masson's trichrome stains. Mouse antiVEGF monoclonal antibody (1:100; Santa Cruz Biotechnology, Santa Cruz, Calif., USA), mouse anti-TSP-1 monoclonal antibody (1:500; Santa Cruz Biotechnology), rabbit anti-HIF-1 $\alpha$ monoclonal antibody (1:100; Santa Cruz Biotechnology), rabbit anti-collagen III polyclonal antibody (1:100; Boster), rabbit anti-CTGF polyclonal antibody (1:50; Boster) and mouse anti-CD34 polyclonal antibody (1:100; Boster) were used for immunohistochemical procedures and the procedures were performed using the SABC Kit and DAB Kit (Zhongshan, Beijing, China) according to manufacturer's instructions. Nonspecific staining was deter- mined by excluding the primary antibodies. Hematoxylin was used for counterstaining procedures. In $10 \times 40$ fields, we randomly selected 10 fields for every section. The integrated optical density (IOD) total of each visual field was determined using the Image 6 Pro Plus system (Media Cybernetics, USA) [8]. IOD represented the quantity of expression of VEGF, CTGF, HIF- $1 \alpha$, collagen III and TSP-1. Under high-power magnification $(\times 400), 10$ randomly selected nonoverlapping fields from the cortical region were analyzed. The fibrotic areas stained in mark blue were picked up on the digital images, and the percentage of the fibrotic area relative to the whole area of the field was calculated; glomeruli and large vessels were not included for image analysis.

\section{Capillary Density}

Tissue samples stained for CD34 expression were examined with light microscopy at $\times 40$ magnification. PTC density was estimated using an adaptation of previously published methods [24]. Capillaries positively stained for CD34 were counted in 10 randomly chosen microscopic fields on each slide, and capillary density was presented as the average number of capillaries $/ 0.065 \mathrm{~mm}^{2}$.

\section{Statistical Analysis}

All statistical analyses were performed using SPSS 13.0 software. Data were expressed as means \pm SD for all experiments. One-way ANOVA was used to assess statistical significance between means. $\mathrm{p}<0.05$ was considered statistically significant.

\section{Results}

\section{Generation of shRNA Vectors for TSP-1 Suppression}

To determine which shRNA vector is most effective, the RMCs were transfected with Thbs1-1, -2 and -3 or control empty vector. The levels of TSP-1 protein expres- 


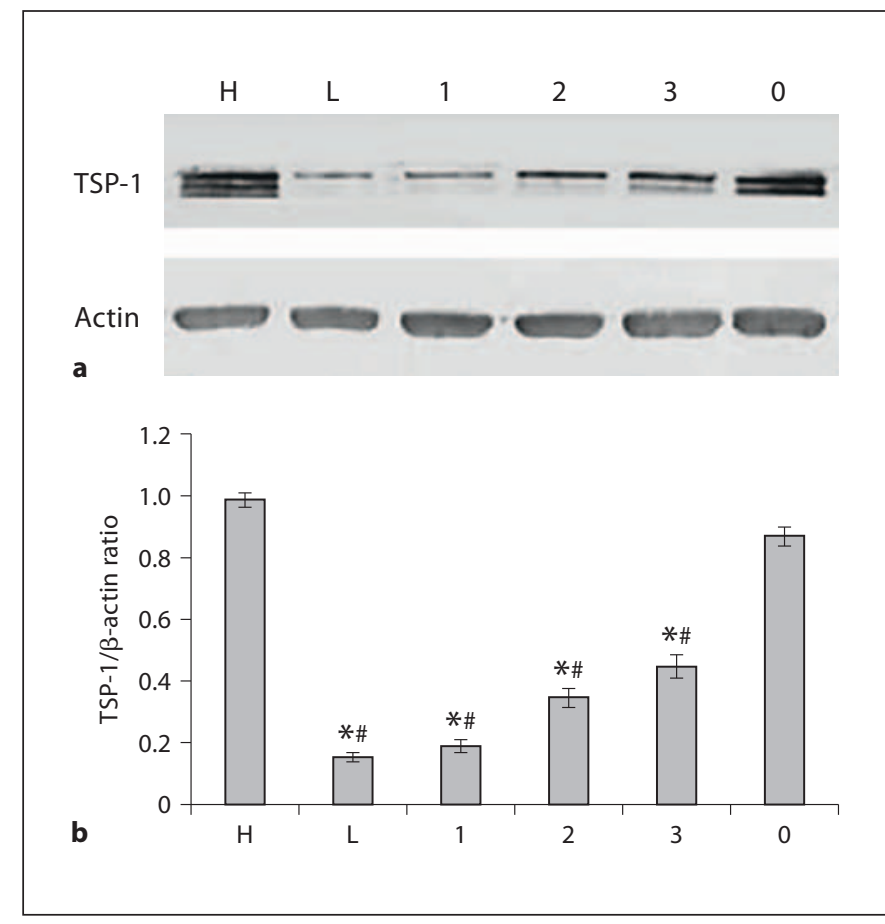

Fig. 1. TSP-1 expression in cultured RMCs after TSP-1 RNAi. RMCs were transfected with Thbs1-1 and cultured in high glucose $(\mathrm{H}, 30 \mathrm{mM})$ or low glucose $(\mathrm{L}, 5.5 \mathrm{mM})$. RMCs were also transfected with Thbs1-1 (1), -2 (2) and -3 (3) or empty vector (0) in high glucose. TSP-1 was maximally induced at a high glucose concentration. a Protein expression of TSP-1 was examined by Western blot analysis. Actin was used as an internal control for quantification. b Western blot analysis shows the shRNA-mediated silencing. The Thbs1-1 significantly inhibited the expression of TSP-1 in RMCs. ${ }^{\#} \mathrm{p}<0.01$ vs. control group (0); ${ }^{*} \mathrm{p}<0.01$ vs. control group $(\mathrm{H})$.

sion and mRNA transcripts were determined by Western blot and RT-PCR, respectively. As shown in figure 1, the expression and reactivity of TSP-1 were maximally induced at a high glucose concentration. Then Thbs1-1, -2 and -3 or control empty vector were transfected with RMCs at a high glucose concentration. Statistically significant interference of TSP-1 expression was achieved by Thbs1-1 (fig. 1). Additionally, the interfering effects of different TSP-1 shRNAs on the expression of TSP-1 protein were observed by RT-PCR (fig. 2). The Thbs1-1 transcript significantly inhibited the expression of TSP-1 in RMCs. Based on its strong interfering activity, Thbs1-1 was further used for the subsequent studies.

TSP-1 shRNA Inhibits the Expression of TSP-1 in UUO

Based on the result of the shRNA interference in cultured RMCs, we probed the effect of TSP-1 shRNA

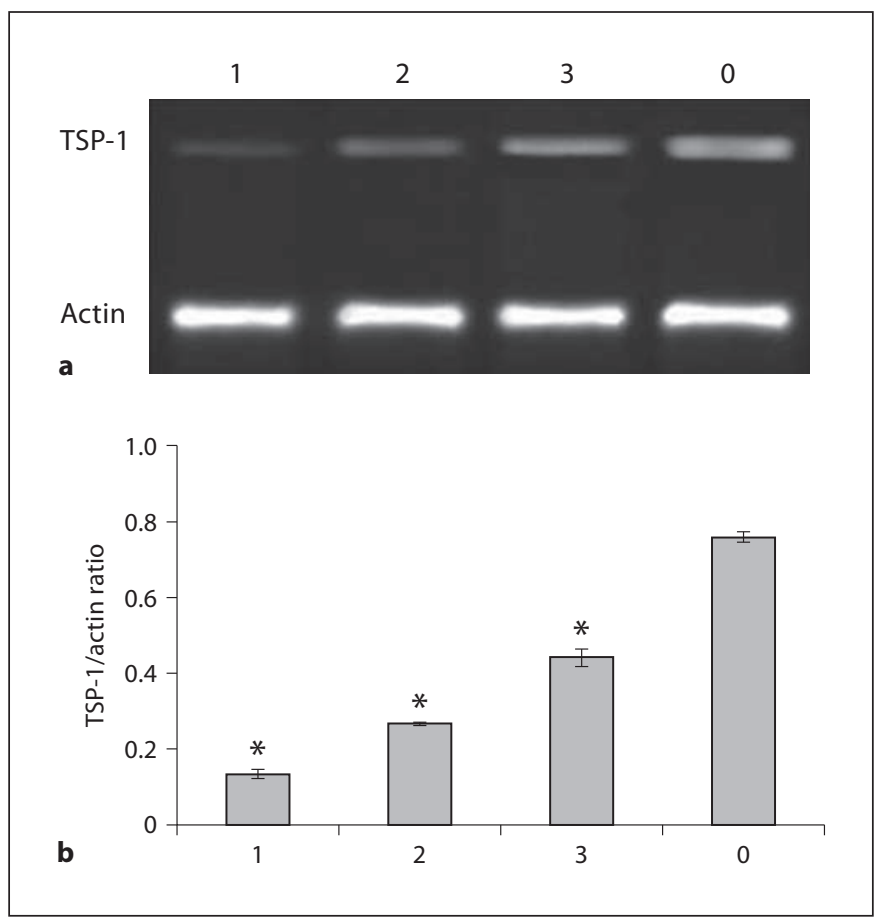

Fig. 2. Effect of shRNA sequence on TSP-1 gene silencing. RMCs were transfected with Thbs1-1 (1), -2 (2) and -3 (3) or empty vector (0). a The mRNA expression of TSP-1 was amplified by RTPCR. Actin was used as an internal control for quantification. b RT-PCR analysis shows the shRNA-mediated silencing, TSP-1 expression in cultured RMCs. Thbs1-1 significantly suppressed the expression of TSP-1. ${ }^{*} \mathrm{p}<0.01$ vs. control group (0).

(Thbs1-1) in UUO kidney in mice. UUO is a well-established experimental model for the study of the mechanisms of renal interstitial fibrosis and the evaluation of potential therapeutic approaches to ameliorate the fibrosis. UUO in mice produces tubulointerstitial inflammation and fibrosis, which appears to simulate the human condition produced by obstructive uropathy [25].

In order to investigate the effect of TSP-1 siRNA (Thbs1-1) in renal fibrosis caused by UUO, we divided mice into four groups: (1) normal control mice (Con), (2) nontreated UUO mice (UUO), (3) UUO mice injected with Thbs1-1 (shRNA) and (4) UUO mice injected with empty vector for ruling out DNA-mediated effects (EV). TSP-1 protein expression was detected by Western blot and immunohistochemistry. TSP-1 protein expression was very low in the Con group (fig. 3c, d). TSP-1 protein was detected at day 1 and progressively increased to day 14 in the UUO and EV groups (fig. 3c, d). In contrast, from day 3 onwards, the expression of TSP-1 was significantly decreased in the shRNA group (fig. $3 c$, d). The im- 


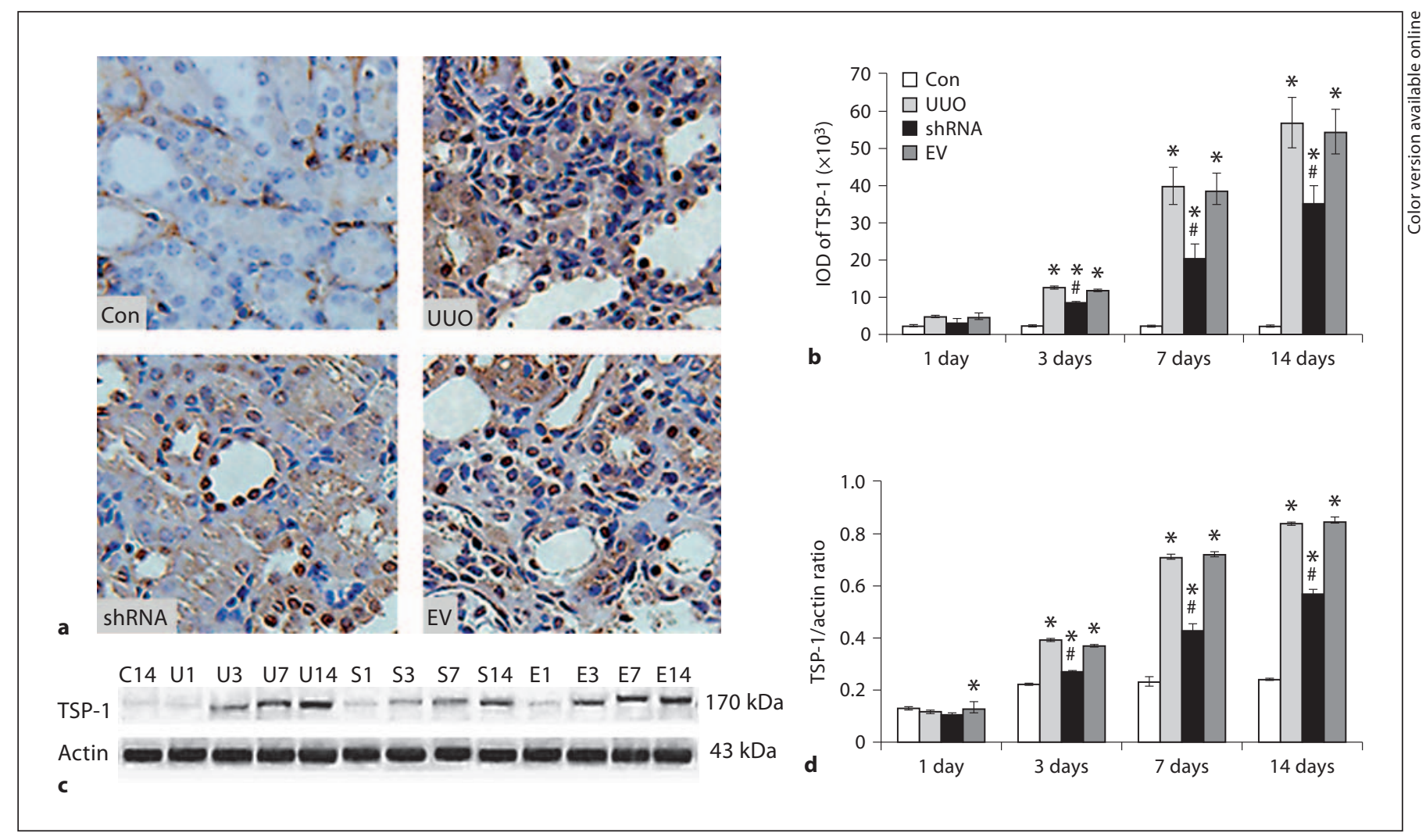

Fig. 3. TSP-1 shRNA attenuated TSP-1 protein in vivo. a TSP-1 staining in tissues samples at day $14(\times 400)$. Little TSP-1 was stained in the Con group. TSP-1 was clearly seen in the cytoplasm of the tubular epithelial cells and tubulointerstitial cells at day 14 in the UUO and EV groups. TSP-1 was stained less in the shRNA group than in the UUO and EV groups. $\mathbf{b}$ Total IOD of TSP-1 in each group; the total IOD in each group represents the protein level. c Western blot of TSP-1 protein expression. C14 represents

munohistochemistry of TSP-1 kidney sections indicated that TSP-1 was clearly observed in the cytoplasm of the tubular epithelial cells and tubulointerstitial cells at day 14 in the UUO and EV groups (fig. 3a), but not detected in the Con group (fig. 3a). However, TSP-1 was stained less at day 14 in the shRNA group than in the UUO and EV groups (fig. 3a). The results show that the level of TSP1 was decreased in the shRNA group at day 3 compared with the UUO group and remained low at days 7 and 14 (fig. 3b), which is in accordance with the results of the Western blot. Taken together, the expression of TSP-1 was suppressed in shThbs1-1-injected UUO kidney in mice.

\section{TSP-1 shRNA Increased VEGF Expression in UUO}

VEGF is a potent endothelial cell mitogen, angiogenic factor and enhancer of vascular permeability. As the Con group at day 14. U1, U3, U7 and U14 represent the UUO group at days 1, 3, 7 and 14, respectively. S1, S3, S7 and S14 represent the shRNA group at days 1, 3, 7 and 14, respectively. E1, E3, E7 and E14 represent the EV group at days 1, 3, 7 and 14, respectively. $\mathbf{d}$ Western blot analysis shows TSP-1 expression in kidney in vivo. Values are means \pm SD. ${ }^{*} p<0.05$ vs. Con group; ${ }^{\#} p<$ 0.05 vs. UUO group. $\mathbf{b}$, $\mathbf{d}$ It can be seen that TSP-1 protein was significantly suppressed in shRNA group.

shown in figure 4a, VEGF was most prominent in glomerular podocytes and in tubular epithelial cells in the Con group. The VEGF staining was gradually reduced, particularly in areas of tubular damage or atrophy, at day 14 in UUO and EV groups (fig. 4a). However, the intensity of VEGF staining was moderate in the tubular epithelial cells in the shRNA group (fig. 4a). The VEGF protein was markedly increased at day 1 in the UUO, EV and shRNA groups, but not in the Con group (fig. 4c, d). There was a significant reduction in VEGF levels in both the UUO and EV groups at days 3, 7 and 14 compared to the Con group, but the VEGF expression was obviously enhanced in mice of the shRNA group compared to mice in the UUO group (fig. $4 \mathrm{c}, \mathrm{d}$ ). The protein expression of VEGF by immunostaining analysis showed similar variation trends with the results of the Western 


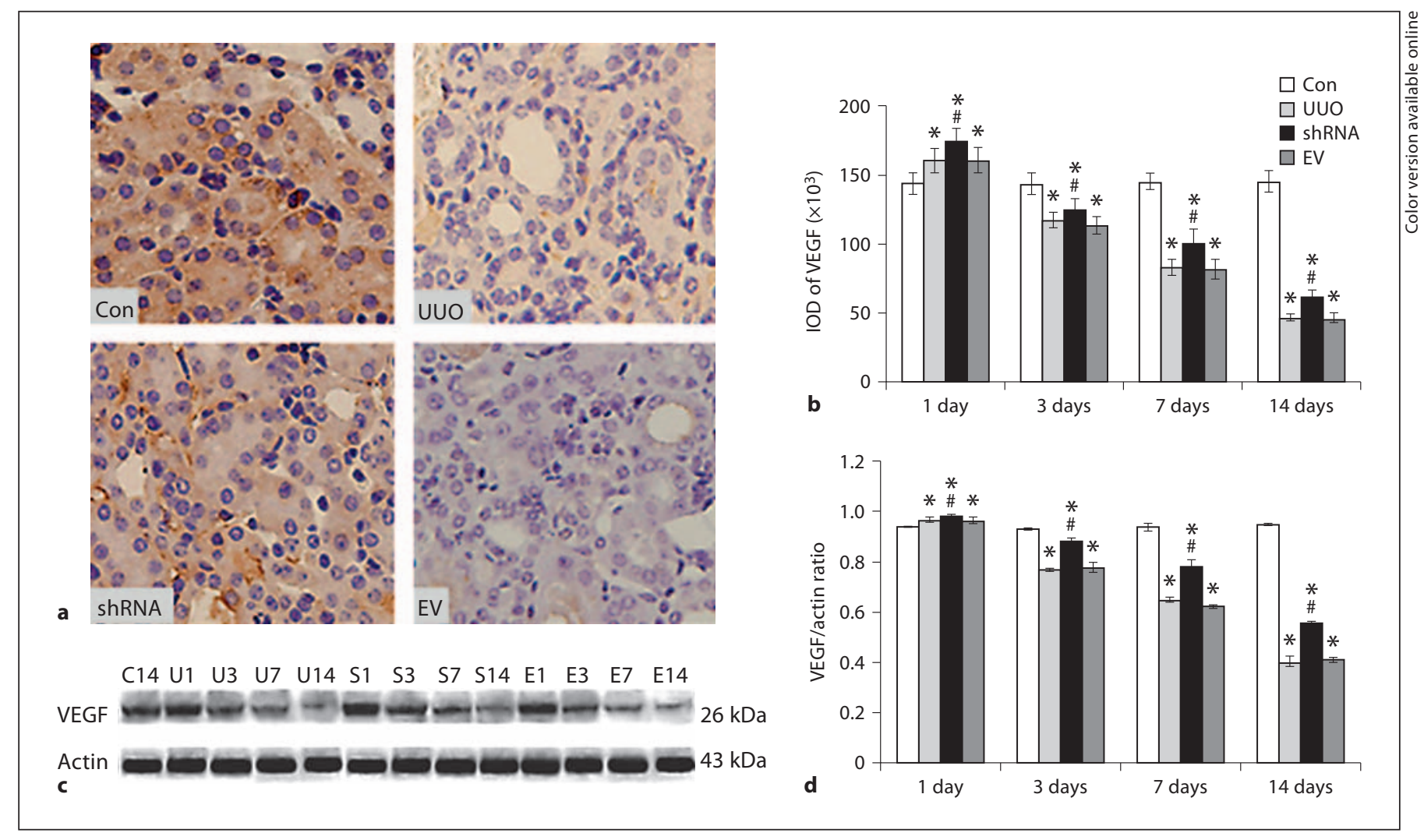

Fig. 4. TSP-1 shRNA increased VEGF expression in UUO. a VEGF staining in tissues samples at day $14(\times 400)$. VEGF was most prominent in the Con group. However, VEGF staining was gradually reduced in the UUO and EV groups. In contrast, the intensity of VEGF staining was moderate in tubular epithelial cells in the shRNA group. $\mathbf{b}$ The total IOD of VEGF in each group; the total IOD in each group represents the protein level. c Western blots of VEGF protein expression. C14 represents the normal control group at day14. U1, U3, U7 and U14 represent the UUO group at days 1, 3, 7 and 14, respectively. S1, S3, S7 and S14 represent the shRNA group at days 1, 3, 7 and 14, respectively. E1, E3, E7 and E14 represent the EV group at days 1, 3, 7 and 14, respectively. d Western blot analysis shows VEGF expression in kidney in vivo. Values are means \pm SD. ${ }^{*} \mathrm{p}<0.05$ vs. Con group; ${ }^{*} \mathrm{p}<0.05$ vs. UUO group. b, $\mathbf{d}$ It can be seen that VEGF protein levels were markedly increased in kidney of UUO mice after Thbs1-1 injection.

nificantly increased at days 7 and 14 (fig. 5b). The results showed that TSP-1 gene silencing alleviated the loss of PTCs in obstructive nephropathy in mice.

\section{TSP-1 shRNA Ameliorated Hypoxia in UUO}

HIF- $1 \alpha$ is a sensitive hypoxia indicator since it is stabilized in a low-oxygen cellular milieu [26]. In order to observe the development of hypoxia, we investigated expression levels of HIF-1 $\alpha$ in renal tissues. In the Con group, there was faint staining of HIF- $1 \alpha$ in cortical nuclei, predominantly in distal tubules (fig. 6a). Nuclear HIF-1 $\alpha$ staining was most apparent in areas of extensive tubular dilatation and interstitial inflammation in the UUO and EV groups at day 14. In these areas, nuclear HIF-1 $\alpha$ staining was seen in both tubular and interstitial 


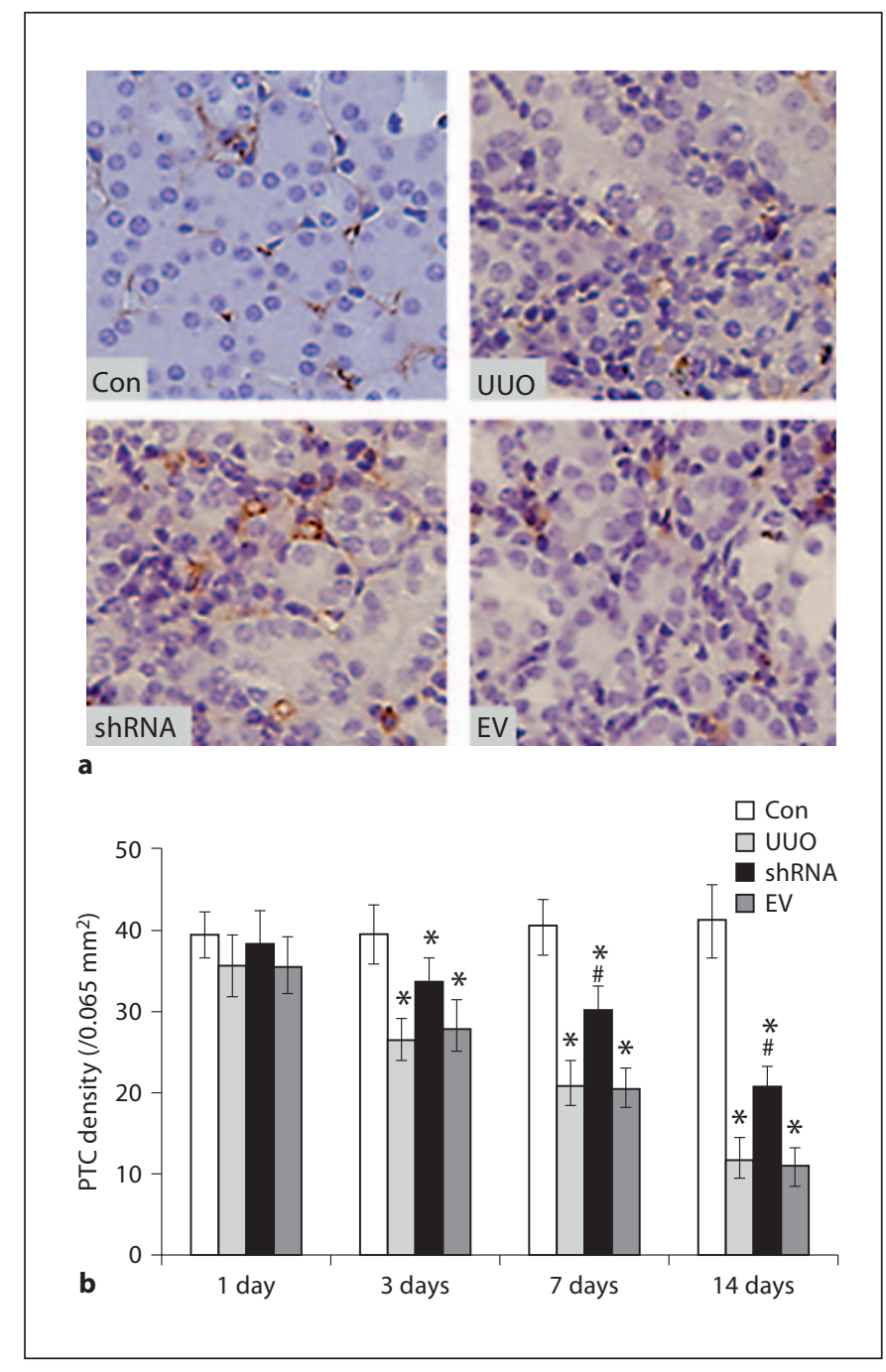

Fig. 5. Changes in PTCs. a PTC staining $(\times 400)$. PTCs of Con group kidneys were patent, displayed uniform size and shape, and were regularly arranged in most of the interstitium. The number of PTCs was obviously decreased in UUO group, especially around the severely atropic tubules and within large areas of interstitial fibrosis. After TSP-1 gene silencing, the number of PTCs in shRNA group was much more than in the UUO and EV groups. b PTC density in each group. It was shown that PTC density was significantly increased after TSP-1 gene silencing. Values are means $\pm \mathrm{SD}$. ${ }^{*} \mathrm{p}<0.05$ vs. Con group; ${ }^{\#} \mathrm{p}<0.05$ vs. UUO group.

cells (fig. 6a). In the shRNA group, HIF-1 $\alpha$ immunostaining was apparently generally decreased in tubules, especially in areas of atrophy and fibrosis at day 14 (fig. 6a). To quantify HIF-1 $\alpha$ levels, Western blot analyses were also done (fig. 6c). There was little HIF-1 $\alpha$ detected in the Con group. In the UUO and EV groups, from day 3 onwards, the expression of HIF-1 $\alpha$ was dramatically increased at the same time-points in comparison to the Con group (fig. 6b, d). However, the level of HIF-1 $\alpha$ protein was decreased in the shRNA group at day 3 compared with the UUO group and remained low at days 7 and 14 (fig. $6 \mathrm{~b}, \mathrm{~d}$ ). The total IOD of HIF-1 $\alpha$ showed a similar reduction trend in HIF-1 $\alpha$ expression in UUO mice after TSP-1 gene silencing. The level of HIF-1 $\alpha$ protein was significantly decreased, and hypoxia was ameliorated in the shRNA group.

\section{TSP-1 shRNA Delays the Process of Fibrosis in vivo}

We investigated fibrosis-induced UUO mice to assess the TSP-1 shRNA effect after Thbs1-1 injection. CTGF is expressed in proliferative and fibrotic glomerular and interstitial lesions of many kinds of human and experimental nephropathies, and is closely related to the degree of renal tubulointerstitial fibrosis [27]. In the Con group, faint CTGF staining could be seen in the glomerular cells and interstitial cells (fig. 7a). However, markedly increased staining of CTGF was noted in the interstitial cells and tubular epithelial cells, interstitial fibrotic regions, and glomerulosclerosis in the UUO and EV groups at day 14 (fig. 7a). In contrast, the staining of CTGF was reduced in renal tissues at day 14 in the shRNA group (fig. 7a). Expression of CTGF began to increase from day 7 in the UUO and EV groups as compared to the Con group, and remained significantly high at day 14 (fig. $7 \mathrm{~b}$ ). Though there was no significant difference in the expression of CTGF at days 1 and 3 among the UUO, EV and shRNA groups, the protein expression level of CTGF in the shRNA group was reduced significantly at days 7 and 14 (fig. 7b). The total IOD of CTGF showed similar variation trends with the results of the Western blot (fig. 7c, d). The expression of CTGF was decreased in Thbs1-1-injected UUO kidney in mice.

We assessed the effects of TSP-1 RNAi on matrix production and renal fibrosis in obstructive nephropathy. As shown in figure 8a, a dramatic expression of collagen III was observed in the obstructed kidney after UUO when compared with the Con group. Treatment with Thbs1-1, however, significantly reduced the collagen deposition (fig. 8b). Administration of Thbs1-1 significantly inhibited type III collagen expression. These results indicate that TSP-1 RNA gene silencing suppresses interstitial matrix overproduction and reduces renal fibrosis in experimental nephropathy in vivo.

No significant histological abnormality and fibrosis were observed in the Con group (fig. 9a, b). Tubular atrophy, infiltration of leukocytes, the expanded tubular interstitial volume and the overaccumulation of the extra- 


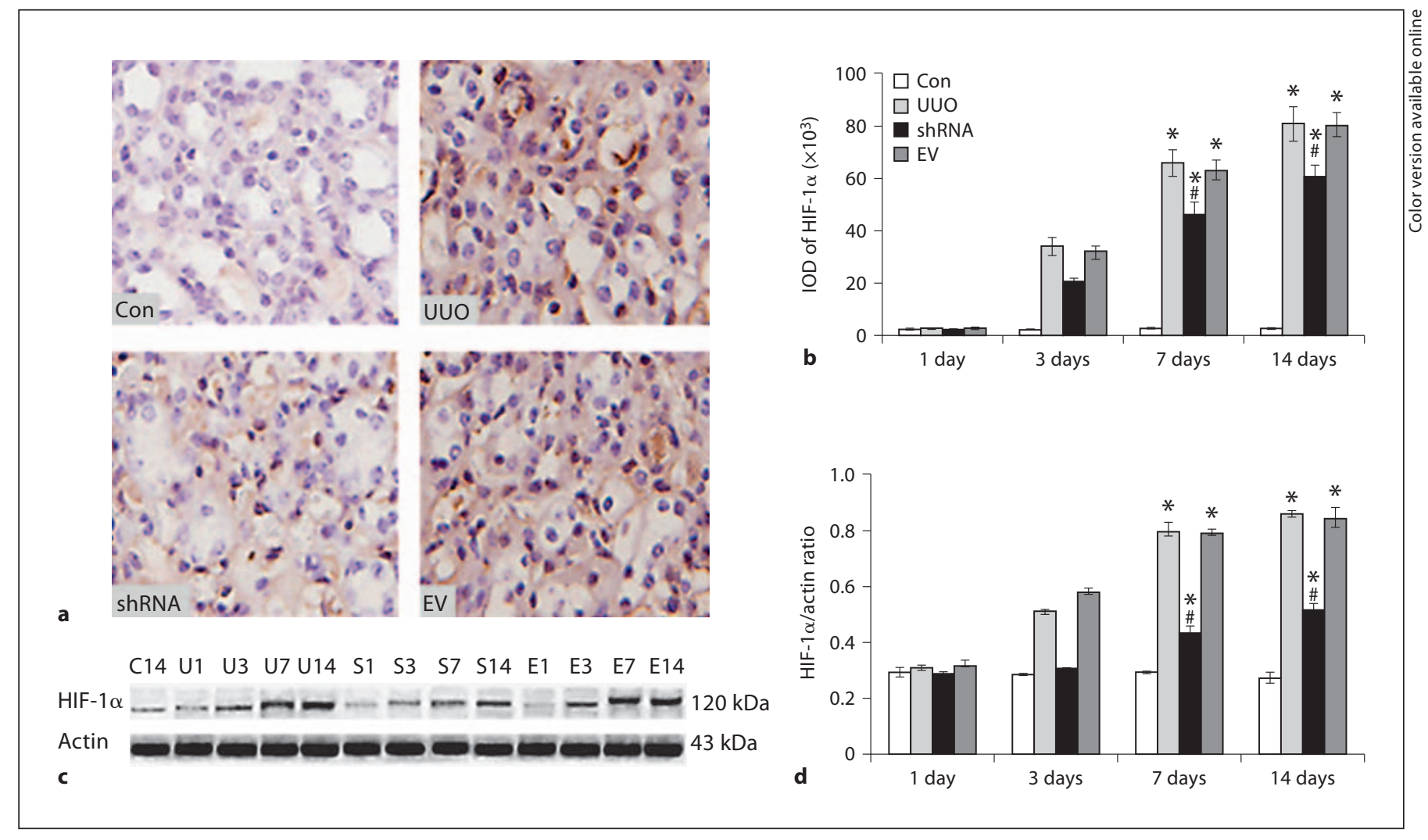

Fig. 6. TSP-1 shRNA ameliorated hypoxia in UUO. a HIF-1 $\alpha$ staining in tissues samples at day $14(\times 400)$. There was faint staining of HIF- $1 \alpha$ in the Con group. Nuclear HIF-1 $\alpha$ staining was most apparent in the UUO and EV groups. In the shRNA group, HIF- $1 \alpha$ immunostaining was apparently decreased in tubules, especially in areas of atrophy and fibrosis. $\mathbf{b}$ The total IOD of HIF$1 \alpha$ in each group; the total IOD in each group represents the protein level. c Western blots of HIF-1 $\alpha$ protein expression. C14 represents the Con group at day 14. U1, U3, U7 and U14 represent the

cellular matrix occurred 7 days after UUO. At day 14, in the UUO and EV groups, almost all of the tubules were destroyed and important renal lesions were observed, including marked interstitial fibrosis and some glomerular sclerosis (fig. 9a, b). The obstructed kidneys of mice in the shRNA group showed fewer destructive changes to the renal tubular structure and less stromal fibrosis and stromal inflammatory cell infiltration than mice in the UUO and EV groups (fig. 9a, b). Fibrosis could be detected in the renal interstitial area at day 7 and was more severe at day 14 when almost all of the tubules were destroyed. The interstitial areas at days 7 and 14 in the shRNA group were markedly lower than in the UUO and EV groups (fig. 9c). TSP-1 gene silencing delayed the development of fibrosis in vivo in the UUO mouse model.
UUO group at days 1, 3, 7 and 14, respectively. S1, S3, S7 and S14 represent the shRNA group at days 1,3,7 and 14, respectively. E1, E3, E7 and E14 represent the EV group at days 1, 3, 7 and 14, respectively. d Western blot analysis shows HIF- $1 \alpha$ expression in kidney in vivo. Values are means $\pm S D .{ }^{*} p<0.05$ vs. Con group; ${ }^{*} \mathrm{p}<0.05$ vs. UUO group. $\mathbf{b}, \mathbf{d}$ It can be seen that TSP-1 gene silencing significantly reduced the expression of HIF-1 $\alpha$ in the kidneys of UUO mice.

\section{Discussion}

The increased TSP-1 expression inhibited both basic fibroblast growth factor- and VEGF-mediated endothelial cell proliferation [28] and independently induced endothelial cell apoptosis [15] in both human and mouse experimental renal fibrosis models $[5,12,29,30]$. TSP-1 plays an important role in the development of fibrosis through activation of TGF- $\beta$ [31]. TSP-1 peptide antagonist could attenuate renal interstitial fibrosis in vivo by preventing TSP-1-mediated TGF- $\beta 1$ activation [32]. TSP-1 is increased in the interstitium and is correlated with the loss of glomerular and peritubular capillaries [5]. There is increasing evidence that the microvasculature plays a critical role in progressive renal disease [5- 


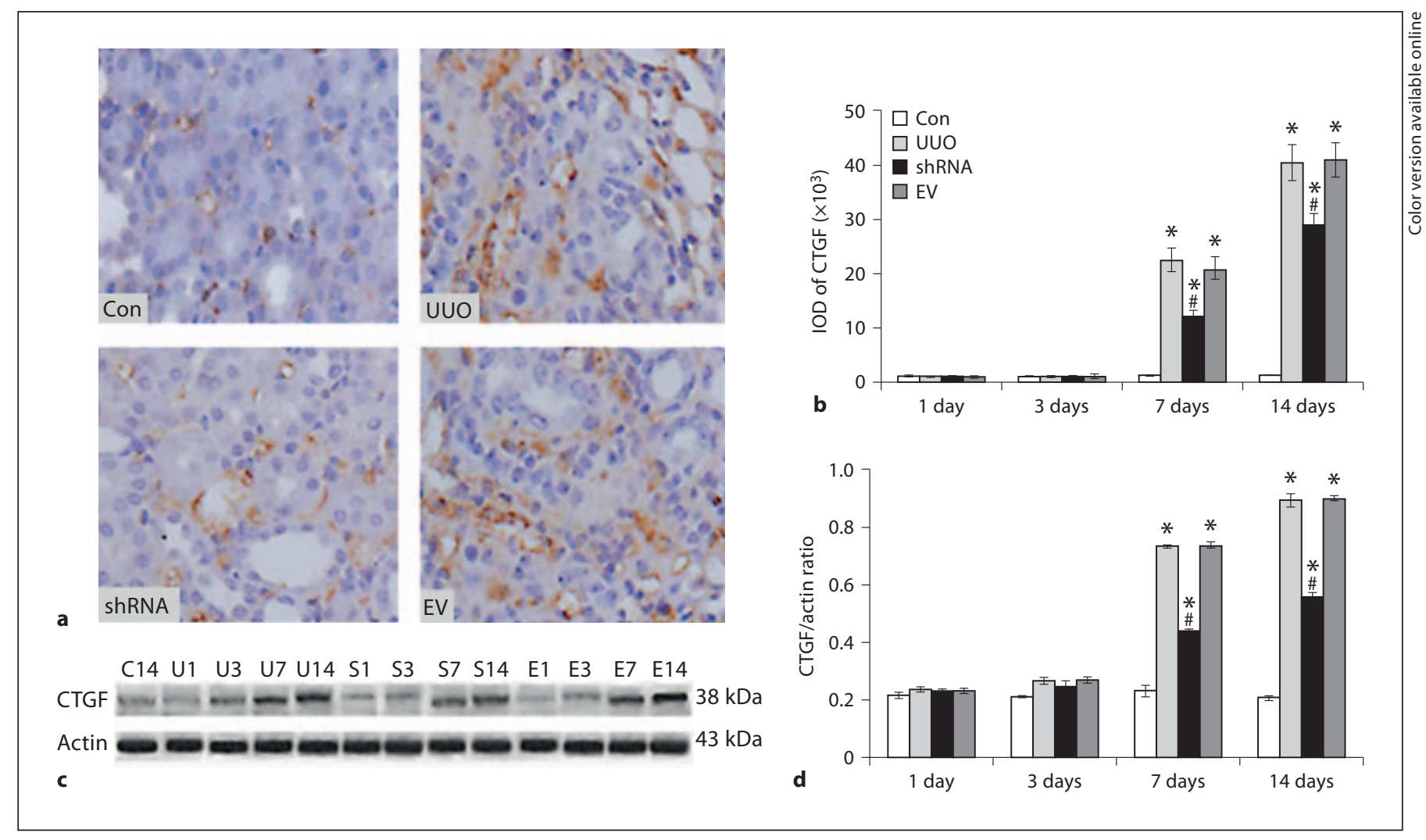

Fig. 7. TSP-1 shRNA delays the process of fibrosis in UUO. a CTGF staining in tissues samples at day $14(\times 400)$. Faint CTGF staining could be seen in the Con group. However, markedly increased staining of CTGF was noted in the UUO and EV groups at day 14. After Thbs1-1 injection, the staining of CTGF was reduced in renal tissues in the shRNA group. $\mathbf{b}$ Total IOD of CTGF in each group; the IOD of each group represents the protein level. c Western blot of CTGF protein expression. C14 represents the
Con group at day 14. U1, U3, U7 and U14 represent the UUO group at days 1, 3, 7 and 14, respectively. S1, S3, S7 and S14 represent the shRNA group at days 1, 3, 7 and 14, respectively. E1, E3, E7 and E14 represent the EV group at days 1, 3, 7 and 14, respectively. d Western blot analysis shows CTGF expression in kidney in vivo. Values are means $\pm \mathrm{SD}$. ${ }^{*} \mathrm{p}<0.05$ vs. Con group; ${ }^{\#} \mathrm{p}<$ 0.05 vs. UUO group. b, d It can be seen that CTGF expression was significantly suppressed after Thbs1-1 injection.
8]. Loss of PTCs would impair delivery of oxygen and nutrients to the tubules and interstitial cells, and then produce chronic ischemia [5]. The present study confirmed our hypothesis that TSP-1 gene silencing could increase PTC density and alleviate the development of renal fibrosis.

There are two general methods of RNAi: one uses chemically synthesized 21-nt siRNA, and the other uses vector-based siRNA (shRNA). shRNA has a more prolonged effect and is relatively inexpensive [33]. We used the shRNA strategy to abolish TSP-1 function and examined the effect on tubulointerstitial fibrosis in vivo. Schwarz et al. [34] hypothesized that RISC preference resulted in the sequence bias between effective and ineffective siRNAs. According to this rule, we used three differ- ent TSP-1 shRNAs to test the efficacy and accuracy of the TSP-1 shRNAs in RMCs in vitro. Only one shRNA, designed Thbs1-1, exhibited the lowest expression of TSP-1 in comparison to the other shRNAs in response to the high glucose concentration at which TSP-1 expression was maximally induced in RMCs. The Thbs1-1 maximally suppressed TSP-1 in RMCs at a high-glucose condition, indicating that the Thbs1-1 contained a good shRNA sequence to inhibit TSP-1 expression.

The effect of Thbs1-1 was examined in vivo in a mouse model of UUO. UUO is a well-established experimental model for the study of the mechanisms of renal interstitial fibrosis and the evaluation of potential therapeutic approaches to ameliorate fibrosis [35]. In vivo studies showed that TSP-1 expression was markedly increased 
from day 3 in the UUO and EV groups. However, Thbs11 significantly suppressed TSP-1 expression from day 3 in the shRNA group. TSP-1 is the first discovered naturally occurring inhibitor of angiogenesis [36]. In order to observe the angiogenesis after Thbs1-1 interference, we detected the expression of VEGF (proangiogenic factor) and PTC density.

VEGF stimulates endothelial cell proliferation and differentiation, increases vascular permeability, mediates endothelium-dependent vasodilatation, and supports vascular survival by preventing endothelial apoptosis [37, 38]. VEGF expression in the UUO, EV and shRNA groups was significantly increased in comparison to the Con group at day 1 , which may be a compensatory response to the body and a period of repair and recovery of microvascular injury [39]. Then we observed that the protein VEGF was markedly reduced as the disease progressed in the UUO and EV groups. In contrast, the VEGF level in the shRNA group was significantly increased compared to the UUO and EV groups at the other three time-points.

We evaluated the amount of PTCs by CD34 immunostaining. In the Con group, it showed no obvious changes in the number of PTCs. PTC injury was gradually aggravated and PTC density was markedly reduced at days 7 and 14 in the UUO and EV groups, respectively. However, PTC density in the shRNA group was significantly increased in comparison to the UUO and EV groups at days 7 and 14. PTC injury is related to a decreased blood supply to renal tubules [40]. The net result of diminished oxygen delivery can lead to further tubulointerstitial injury [36]. After TSP-1 gene silencing, the injury of PTC density was dramatically ameliorated in UUO mice in this study.

To observe development of hypoxia in mice of the UUO group, we investigated the expression of HIF1- $\alpha$. HIF-1 $\alpha$ is the master hypoxia response regulator, the amount of which is determined by oxygen-dependent degradation [41]. HIF-1 $\alpha$ protein was relatively low in the Con group, but its expression gradually increased in the UUO and EV groups. In the shRNA group, the expression of HIF-1 $\alpha$ was markedly reduced in comparison to the UUO and EV groups at days 7 and 14, respectively. Hypoxia was ameliorated after TSP-1 shRNA interference.

In order to observe the fibrosis of kidney in UUO, we detected the level of CTGF, collagen III and areas of tubulointerstitial fibrosis. CTGF was shown to induce kidney fibroblast proliferation and extracellular matrix synthesis [42]. A local increase of CTGF expression may play a role in the development of glomerulosclerosis and tubulointerstitial fibrosis [26]. Collagen III is markedly accu-

mulated in the pathogenesis of renal interstitial fibrosis by UUO [43]. The expression of CTGF and collagen III was significantly increased in the UUO and EV groups in this study. However, the expression of CTGF and collagen III in the shRNA group was markedly reduced in comparison to the UUO and EV groups at days 7 and 14. From the morphologic data, we found that the development of

Fig. 8. TSP-1 shRNA delays collagen III expression in the kidneys of UUO mice. a Collagen III staining in tissue samples at day 14 $(\times 400)$. Collagen III staining was faint in the Con group; however, markedly increased staining of collagen III was noted in the UUO and EV groups at day 14. After Thbs1-1 injection, the staining of collagen III was reduced in the renal tissues of the shRNA group. b Total IOD of collagen III in each group; the IOD of each group represents the protein levels. Values are means $\pm \mathrm{SD} .{ }^{*} \mathrm{p}<$ 0.05 vs. Con group; ${ }^{\#} \mathrm{p}<0.05$ vs. UUO group. 


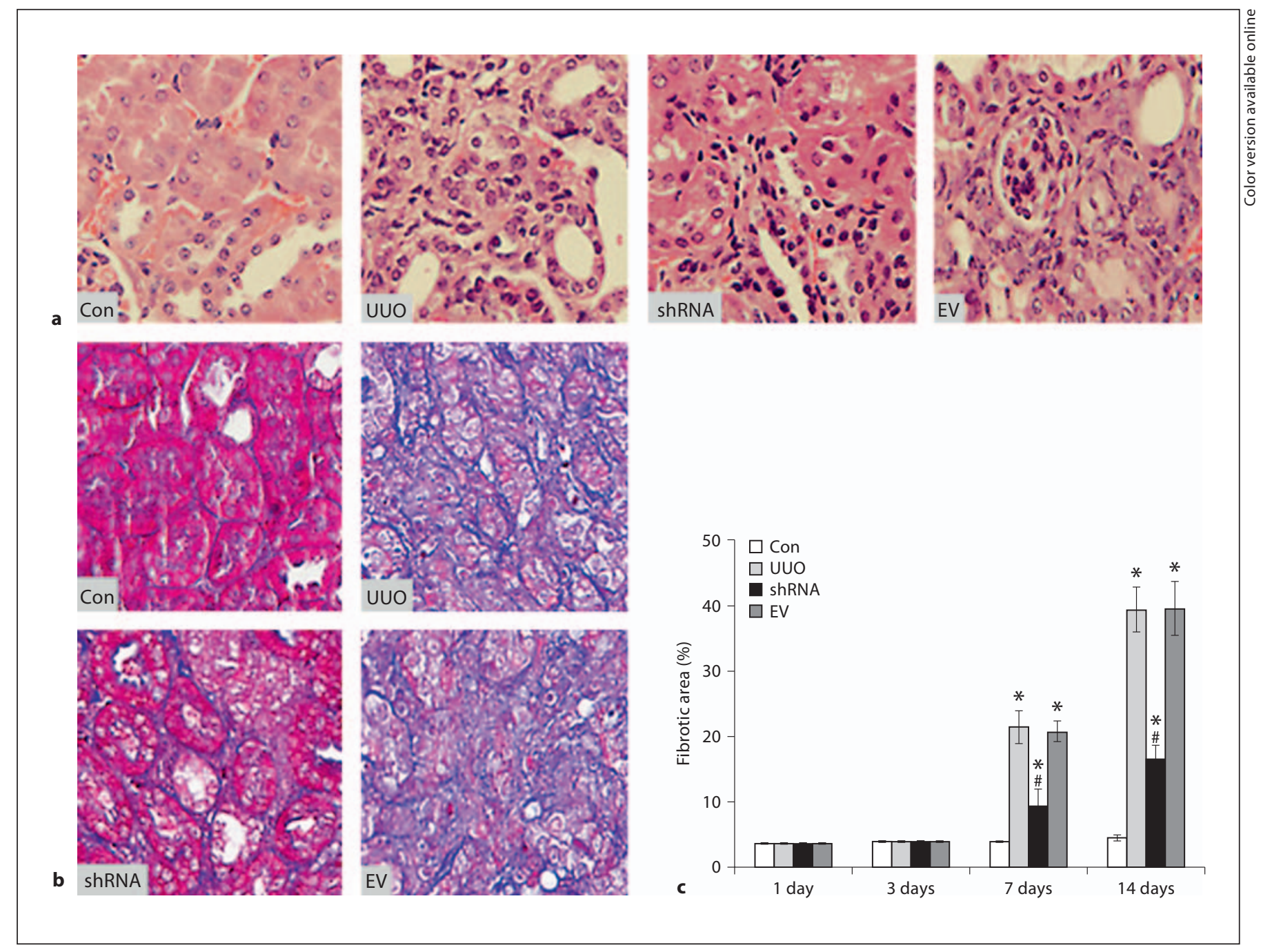

Fig. 9. Representative images of histological features $(\times 400)$. a HE staining of tissue samples at day 14 . There was no significant histological abnormality in the Con group. Almost all of the tubules were destroyed and important renal lesions were observed in the UUO and EV groups. The obstructed kidneys of mice in the shRNA group showed fewer destructive changes to the renal tubular structure. b Masson staining of tissue samples at day 14 .
Renal interstitial fibrosis was obvious in the UUO and EV groups. After Thbs1-1 injection, renal interstitial fibrosis was weaker in the shRNA group than in the UUO and EV groups. $c$ The areas of renal interstitial fibrosis in each group. TSP-1 shRNA interference significantly inhibited the development of tubulointerstitial fibrosis. Values are means $\pm \mathrm{SD} .{ }^{*} \mathrm{p}<0.05$ vs. Con group; ${ }^{\#} \mathrm{p}<$ 0.05 vs. UUO group. renal interstitial fibrosis was delayed after TSP-1 injection in UUO mice.

Following the upregulation of TSP- 1 and the reduction of VEGF, PTC density was reduced and HIF- $1 \alpha$ was increased, and the tubulointerstitial fibrosis area was increased after UUO in mice. Microvascular injury, with consequent tissue hypoxia and ischemia, may play an important role in obstructive nephropathy. After TSP-1 shRNA interference, TSP-1 expression was suppressed, VEGF protein expression and PTC density were in- creased, HIF- $1 \alpha$ and CTGF expression were reduced, and renal interstitial fibrosis was alleviated. TSP-1 shRNA gene silencing alleviated renal interstitial fibrosis in the UUO mouse.

In conclusion, we found that Thbs1-1 was a unique and effective sequence for TSP-1 RNAi. Using Thbs1-1, we constructed a shRNA expression vector for TSP-1 interference. Thbs1-1 alleviated UUO-induced fibrosis through the suppression of TSP-1, the increase in VEGF levels and PTC density, and the amelioration of hypoxia in UUO 
mice. Therefore, these findings suggested that TSP-1 gene silencing increased PTC density and prevented renal fibrosis. TSP-1 gene silencing may be a new potential therapeutic option or supplemental therapy for renal fibrosis.
Xuzhou Medical College, Qinglan Lan Project (53051106); Special Talents Fund of Dean of Xuzhou Medical College (2010KJZ23); the Project of Technology Development Foundation of Xuzhou City (XF10C76); and the Project of 7th 'Liu Da Ren Cai Gao Feng' of Jiangsu Province, China (2010-ws043).

\section{Acknowledgments}

This work was supported by the Fund of the Educational Science Foundation of Jiangsu Province, China (No. 07KJB320121); the Project of the Science Foundation of Affiliated Hospital of

\section{Disclosure Statement}

All authors declare they have no competing interests.

\section{References}

1 Chevalier RL, Forbes MS, Thornhill BA: Ureteral obstruction as a model of renal interstitial fibrosis and obstructive nephropathy. Kidney Int 2009;75:1145-1152.

$\checkmark 2$ Nath KA: Tubulointerstitial changes as a major determinant in the progression of renal damage. Am J Kidney Dis 1992;20:1-17.

$\checkmark 3$ Risdon RA, Sloper JC, De Wardener HE: Relationship between renal function and histological changes found in renal-biopsy specimens from patients with persistent glomerular nephritis. Lancet 1968;2:363-366.

$\checkmark 4$ Bohle A, Mackensen-Haen S, Wehrmann M: Significance of post-glomerular capillaries in the pathogenesis of chronic renal failure. Kidney Blood Press Res 1996;19:191-195.

$\checkmark 5$ Kang DH, Joly AH, Oh SW, Hugo C, Kerjaschki D, Gordon KL, Mazzali M, Jefferson JA, Hughes J, Madsen KM, Schreiner GF, Johnson RJ: Impaired angiogenesis in the remnant kidney model. I. Potential role of vascular endothelial growth factor and thrombospondin-1. J Am Soc Nephrol 2001; 12:1434-1447.

6 Kang DH, Hughes J, Mazzali M, Schreiner GF, Johnson RJ: Impaired angiogenesis in the remnant kidney model. II. Vascular endothelial growth factor administration reduces renal fibrosis and stabilizes renal function. J Am Soc Nephrol 2001;12:1448-1457.

$\checkmark 7$ Kang DH, Kanellis J, Hugo C, Truong L, Anderson S, Kerjaschki D, Schreiner GF, Johnson RJ: Role of the microvascular endothelium in progressive renal disease. J Am Soc Nephrol 2002;13:806-816.

$>8$ Sun D, Feng J, Dai C, Sun L, Jin T, Ma J, Wang L: Role of peritubular capillary loss and hypoxia in progressive tubulointerstitial fibrosis in a rat model of aristolochic acid nephropathy. Am J Nephrol 2006;26:363-371.

$\checkmark 9$ Fine LG, Orphanides C, Norman JT: Progressive renal disease: the chronic hypoxia hypothesis. Kidney Int Suppl 1998;65:S74S78.

10 Chen H, Herndon ME, Lawler J: The cell biology of thrombospondin-1. Matrix Bio 2000;19:597-614
11 Abrass CK, Adcox MJ, Raugi GJ: Aging-associated changes in renal extracellular matrix. Am J Pathol 1995;146:742-752.

-12 Hugo C, Shankland SJ, Pichler RH, Couser WG, Johnson RJ: Thrombospondin 1 precedes and predicts the development of tubulointerstitial fibrosis in glomerular disease in the rat. Kidney Int 1998;53:302-311.

13 McGregor B, Colon S, Mutin M, Chignier E, Zech P, McGregor J: Thrombospondin in human glomerulopathies. A marker of inflammation and early fibrosis. Am J Pathol 1994; 144:1281-1287.

14 Guo N, Krutzsch HC, Inman JK, Roberts DD: Thrombospondin 1 and type I repeat peptides of thrombospondin 1 specifically induce apoptosis of endothelial cells. Cancer Res 1997;57:1735-1742.

15 Zhang X, Lawler J: Thrombospondin-based antiangiogenic therapy. Microvasc Res 2007; 74:90-99.

16 Kaihatsu K, Braasch DA, Cansizoglu A, Corey DR: Enhanced strand invasion by peptide nucleic acid-peptide conjugates. Biochemistry 2002;41:11118-11125.

17 Bertrand JR, Pottier M, Vekris A, Opolon P, Maksimenko A, Malvy C: Comparison of antisense oligonucleotides and siRNAs in cell culture and in vivo. Biochem Biophys Res Commun 2002;296:1000-1004.

18 Stewart CK, Li J, Golovan SP: Adverse effects induced by short hairpin RNA expression in porcine fetal fibroblasts. Biochem Biophys Res Commun 2008;370:113-117.

$\checkmark 19$ McAnuff MA, Rettig GR, Rice KG: Potency of siRNA versus shRNA mediated knockdown in vivo. J Pharm Sci 2007;96:29222930

-20 Siolas D, Lerner C, Burchard J, Ge W, Linsley PS, Paddison PJ, Hannon GJ, Cleary MA: Synthetic shRNAs as potent RNAi triggers. Nat Biotechnol 2005;23:227-231.

-21 Vlassov AV, Korba B, Farrar K, Mukerjee S, Seyhan AA, Ilves H, Kaspar RL, Leake D, Kazakov SA, Johnston BH: shRNAs targeting hepatitis C: effects of sequence and structural features, and comparision with siRNA. Oligonucleotides 2007;17:223-236.
22 Masaki T, Foti R, Hill PA, Ikezumi Y, Atkins RC, Nikolic-Paterson DJ: Activation of the ERK pathway precedes tubular proliferation in the obstructed rat kidney. Kidney Int 2003;63:1256-1264.

23 Hwang M, Kim HJ, Noh HJ, Chang YC, Chae YM, Kim KH, Jeon JP, Lee TS, Oh HK, Lee YS, Park KK: TGF-betal siRNA suppresses the tubulointerstitial fibrosis in the kidney of ureteral obstruction. Exp Mol Pathol 2006; 81:48-54.

24 Kairaitis LK, Wang Y, Gassmann M, Tay YC, Harris DC: HIF-1alpha expression follows microvascular loss in advanced murine adriamycin nephrosis. Am J Physiol Renal Physiol 2005;288:F198-F206.

25 Hruska KA: Treatment of chronic tubulointerstitial disease: a new concept. Kidney Int 2002;61:1911-1922.

26 Stravodimos KG, Koritsiadis G, Lazaris AC: Hydronephrosis promotes expression of hypoxia-inducible factor $1 \alpha$. Urol Int 2009; 82:38-42.

27 ItoY, Aten J, Bende RJ, Oemar BS, Rabelink TJ, Weening JJ, Goldschmeding R: Expression of connective tissue growth factor in human renal fibrosis. Kidney Int 1998;53:853861 .

28 Iruela-Arispe ML, Bornstein P, Sage $\mathrm{H}$ : Thrombospondin exerts an antiangiogenic effect on cord formation by endothelial cells in vitro. Proc Natl Acad Sci USA 1991;88: 5026-5030.

-29 Hugo C, Kang DH, Johnson RJ: Sustained expression of thrombospondin-1 is associated with the development of glomerular and tubulointerstitial fibrosis in the remnant kidney model. Nephron 2002;90:460-470.

30 Kang DH, Anderson S, Kim YG, Mazzalli M, Suga S, Jefferson JA, Gordon KL, Oyama TT, Hughes J, Hugo C, Kerjaschki D, Schreiner GF, Johnson RJ: Impaired angiogenesis in the aging kidney: vascular endothelial growth factor and thrombospondin-1 in renal disease. Am J Kidney Dis 2001;37:601611. 
-31 Schultz-Cherry S, Ribeiro S, Gentry L, Murphy-Ullrich JE: Thrombospondin binds and activates the small and large forms of latent transforming growth factor-beta in a chemically defined system. J Biol Chem 1994;269: 26775-26782.

>32 Xie XS, Li FY, Liu HC, Deng Y, Li Z, Fan JM: LSKL, a peptide antagonist of thrombospondin-1, attenuates renal interstitial fibrosis in rats with unilateral ureteral obstruction. Arch Pharma Res 2010;33:275-284.

-33 Brummelkamp TR, Bernards R, Agami R: A system for stable expression of short interfering RNAs in mammalian cells. Science 2002; 296:550-553.

-34 Schwarz DS, Hutvágner G, Du T XZ, Aronin N, Zamore PD: Asymmetry in the assembly of the RNAi enzyme complex. Cell 2003;115: 199-208.
35 Klahr S, Morrissey J: Obstructive nephropathy and renal fibrosis. Am J Physiol Renal Physiol 2002;283:F861-F875.

36 Good DJ, Polverini PJ, Rastinejad F, Le Beau MM, Lemons RS, Frazier WA, Bouck NP: A tumor suppressor-dependent inhibitor of angiogenesis is immunologically and functionally indistinguishable from a fragment of thrombospondin. Proc Natl Acad Sci USA 1990;87:6624-6628.

37 Ferrara N, Gerber HP: The role of vascular endothelial growth factor in angiogenesis. Acta Haematol 2001;106:148-156.

38 Neufeld G, Cohen T, Gengrinovitch S, Poltorak Z: Vascular endothelial growth factor (VEGF) and its receptors. FASEB J 1999;13 9-22.

39 Wakelin SJ, Marson L, Howie SE: The role of vascular endothelial growth factor in the kidney in health and disease. Nephron Physiol 2004;98:p73-p79.
40 Choi YJ, Chakraborty S, Nguyen V: Peritubular capillary loss is associated with chronic tubulointerstitial injury in human kidney: altered expression of vascular endothelial growth factor. Hum Pathol 2000;31:14911497.

41 Safran M, Kaelin WG Jr: HIF hydroxylation and the mammalian oxygen-sensing pathway. J Clin Invest 2003;111:779-783.

42 Frazier K, Williams S, Kothapalli D, Klapper $\mathrm{H}$, Grotendorst GR: Stimulation of fibroblast cell growth, matrix production, and granulation tissue formation by connective tissue growth factor. J Invest Dermatol 1996;107: 404-411.

43 Li Y, Tan X, Dai C, Stolz DB, Wang D, Liu Y: Inhibition of integrin-linked kinase attenuates renal interstitial fibrosis. J Am Soc Nephrol 2009;20:1907-1918. 